\title{
Scheduling Optimization for M2M Communications in LTE-M
}

\author{
SAMIR DAWALIBY, ABBAS BRADAI and YANNIS POUSSET \\ Affiliation: University of Poitiers - XLIM, Poitiers, France \\ samirdawaliby@gmail.com, \{abbas.bradai, yannis.pousset\}@univ-poitiers.fr
}

\begin{abstract}
One of the major challenges for Machine-toMachine (M2M) paradigm is the capability to scale to a larger number of connected devices and to provide ultra-low delay communications in order to meet future Internet of Things (IoT) requirements. In this paper, we study the performance of LTE-M protocol in terms of delay, throughput and bandwidth utilization. Next, we propose a novel scheduling mechanism for LTE-M protocol to support M2M communications. The ultimate goal is to optimally allocate the resource blocks to different users in a more efficient manner in order to maximize the usage of the available bandwidth and reduce communications delay.
\end{abstract}

\section{INTRODUCTION}

In the era of the Internet of Things (IoT), tens of billions of humans and physical objects will communicate simultaneously through wireless heterogeneous networks. However, current networks infrastructures are not capable yet to meet machineto-machine (M2M) communication features which demands the deployment of low-cost machine-type devices (MTDs), lowpowered in terms of energy and low-delay to support critical control applications [1]. Moreover, M2M includes various application areas such as telemetry, remote monitoring and product restocking are all integrated into the IoT. Hence, future $5 \mathrm{G}$ wireless networks will include a mixture of interconnected communication standards and radio access technologies on the same network platform [2]. LTE-M is one of the candidate's protocols which expands LTE for machines and allows long distance communications between MTDs through cellular networks. Although LTE-M supports secure, energy-efficient and cost-effective communications for low-end devices, many enhancements and modifications in terms of protocol still need to be applied.

In this paper, we evaluate the performance of LTE-M in terms of bandwidth and delay and we propose a scheduling optimization approach which increases the number of connected MTDs and supports better QoS for real-time applications such as mobile health (M-Health) that demands extremely low latencies on the order of $1 \mathrm{~ms}$ accuracy [3].

The rest of the paper is structured as follows: Section II presents the global architecture and an outline of LTE-M in a M2M uplink scenario. Section III lists LTE-M advantages and shortcomings in M2M based on NS3 network simulation results and compared to the expected network requirements for the IoT. Our proposed approach and future work are presented in Section IV and Section V concludes the paper.

\section{LTE-M ARCHITECTURE IN UPLINK}

Based on the LTE-M network architecture proposed by EXALTED Project [4], Figure 1 illustrates an uplink scenario in which LTE-M devices (sensors, smart meters, actuators, etc.) upload data traffic to the remote servers with a transmission power of $20 \mathrm{dBm}$. Moreover, Friis Free-space is considered as a propagation loss model with the objective to reach ideal results. Each LTE-M device operates as a standalone device, communicates with other devices over the LTE-M network, or can form a capillary network with other devices of the same type and connects with the external network via a more powerful LTE-M device acting as gateway. The goal of this M2M gateway is to perform protocol translation and data aggregation before transmitting messages to the application server via a specific LTE-M interface.

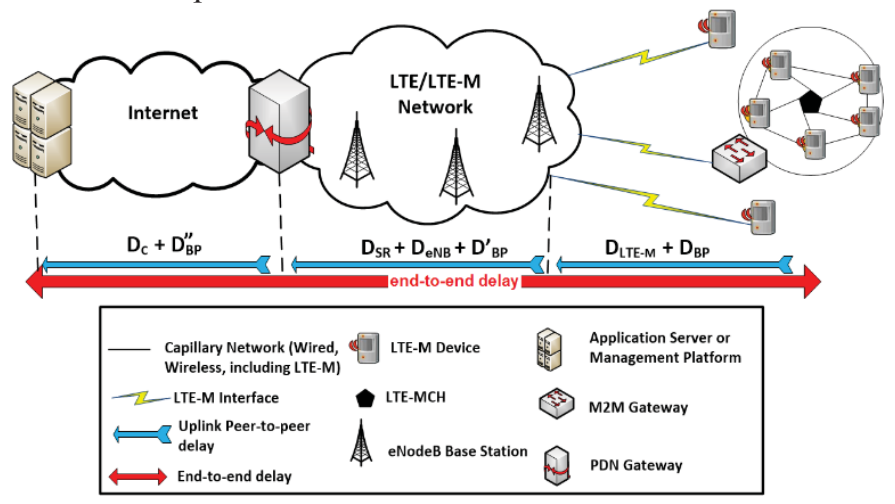

Fig. 1. LTE/LTE-M Network Architecture

During uplink transmission, the generated end-to-end delay is distributed between LTE network components. $\mathrm{D}_{\mathrm{BP}}$ stands for buffering and propagation time delay during uplink and differs depending on the sending node ( $\mathrm{D}_{\mathrm{BP}}, \mathrm{D}^{\prime}{ }_{\mathrm{BP}}$ and $\left.\mathrm{D}{ }^{\prime}{ }_{\mathrm{BP}}\right)$. The LTE-M delay ( $\mathrm{D}_{\mathrm{LTE}-\mathrm{M}}$ ) produced by the LTE-M device is added to $\left(\mathrm{D}_{\mathrm{BP}}\right)$ to bring out the delay that happens between the eNodeB and the LTE-M device. Next, $\left(\mathrm{D}_{\mathrm{SR}}\right)$ is added to (D' $\left.{ }_{\mathrm{BP}}\right)$ and stands for the scheduling process delay caused by the eNodeB at the MAC-layer level and HARQ packet retransmissions delay. And finally, (D' ${ }_{\mathrm{BP}}$ ) propagation delay is added to (Dc), the delay that happens on the core network side.

\section{LTE-M Advantages And SHORTCOMIngs For M2M COMMUNICATIONS}

With LTE-M, physical objects are capable of communicating between each other for longer distances through already existing LTE cellular network. Hence, maintaining older base stations instead of building new ones would be an easier task for mobile operators. Based on our network simulation carried out through the LTE module of NS3 Simulator, LTE-M offers due to its small bandwidth (1.4 MHz with 6 Resource Blocks (RBs) per subframe) a large coverage area. In addition, a single LTE-M device moving away from the eNodeB can upload stable data traffic with a maximum throughput of $1 \mathrm{Mbps}$ for a distance of $13 \mathrm{KM}$ as shown in Figure 2 below. However, one shortcoming of LTE-M is throughput that still needs to be reduced because in many applications, the data message can be 
less than 1 Byte. Moreover, Figure 2 also illustrates end-to-end delay in uplink traffic which varies between 15 and $30 \mathrm{~ms}$ for the first $13 \mathrm{KM}$ which means that LTE-M do not deliver reduced latencies lower than $10 \mathrm{~ms}$ [5] required for future $5 \mathrm{G}$ real-time applications such as robotics, remote conference, etc.

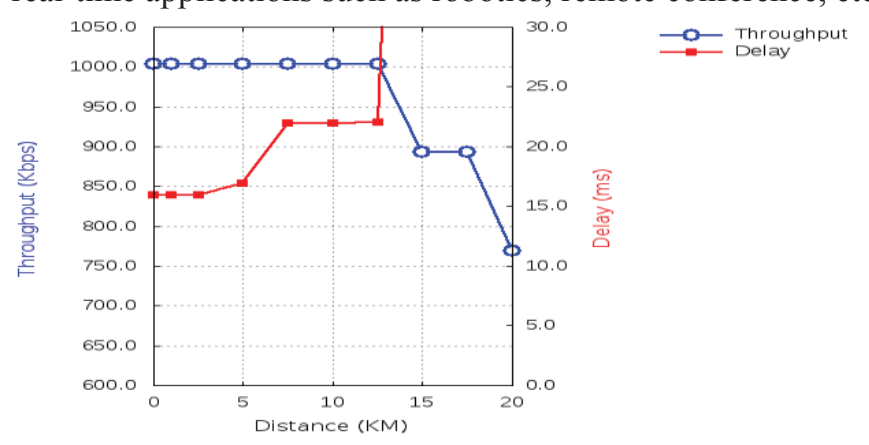

Fig. 2. Throughput and Delay Variation with Distance

Furthermore, in a congestion scenario, the uplink mean throughput transmitted by LTE-M devices is affected by the chosen scheduling methodology as shown in Figure 3 below. Three scheduling algorithms are considered: Proportional Fair (PF), Maximum Throughput (MT) and Round Robin (RR).

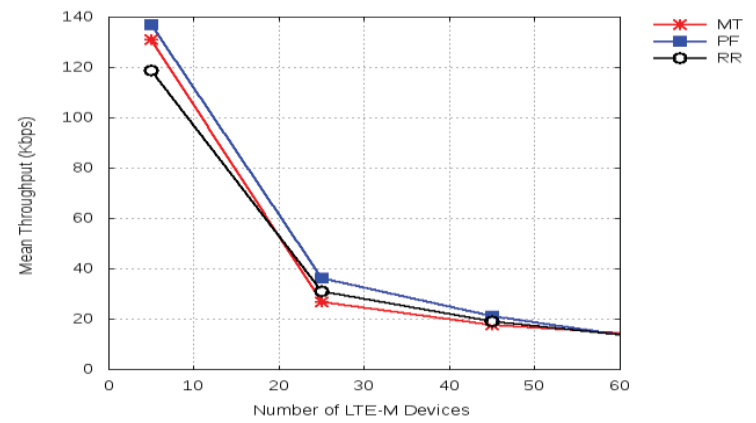

Fig. 3. Mean Throughput Variation with Scheduling Algorithms

Among the three scheduling algorithms, PF shows the best results because it takes into consideration the QoS and provides a fair chance for LTE-M devices to be scheduled. Hence, scheduling prioritization is maintained for devices with good channel condition. RR had the lowest throughput when the number of connected devices was less than 23 because it does not consider the channel condition of LTE-M devices during scheduling process. The worst mean throughput is reached with MT scheduler when the number of devices was higher than 23, this is because MT is a QoS unaware scheduler and only consider devices with good channel conditions. Thus, when the number of devices increase, network congestion will degrade channel conditions and decrease the mean throughput.

\section{THE PROPOSED APPROACH}

Our scheduling approach aims to dynamically schedule RBs at a specific time while achieving a maximum utility for M2M devices. The number of RBs allocated to a device is selected in a way that reduces the communication latency and increases the bandwidth utilization while taking into consideration the device quality of service level. Thus, services with lowest latency are scheduled and served first. In this RBs affectation solution, we define $V_{i}^{j}$ (i for lines and $j$ for columns), a Boolean variable that indicates whether the selected RB is affected or not:

\section{$V_{i}^{j}\left\{\begin{array}{l}1 \text { if the } R B \text { is affected by a M2M device } \\ 0 \text { Otherwise }\end{array}\right.$}

$\mathrm{U}_{\mathrm{k}}$ stands for the service utility in a way that the lower $\mathrm{k}$ index, the more reduced delay is required by the service with the larger service utility value. In this mechanism, the scheduler chooses the number of affected resource blocks per user while respecting the following conditions:

Subject to

$$
\operatorname{Max} \sum_{i=1}^{n} \sum_{j=1}^{n} V_{i}^{j} \cdot U_{k}
$$

And

$$
\left.\sum_{j=1}^{n} V_{i}^{j}=1 \text { (with } i \text { constant }\right)
$$

$$
\sum_{i=1}^{n} V_{i}^{j} \leq n(\text { with } j \text { constant })
$$

Where $\mathrm{n}$ is the number of devices

$\mathrm{D}$ is the M2M device to be scheduled

$\mathrm{RB}$ the resource block

The mapping between M2M devices and RBs are placed with the knapsack capacity as illustrated in Table 1 below.

\begin{tabular}{|c|c|c|c|c|c|}
\hline & $D_{1}$ & $D_{2}$ &.. & $D_{n-1}$ & $D_{n}$ \\
\hline$R B_{1}$ & $V_{1}^{1}$ & $V_{1}^{2}$ &.. & $V_{1}^{n-1}$ & $V_{1}^{n}$ \\
\hline$R B_{2}$ & $V_{2}^{1}$ & $V_{2}^{2}$ &.. & $V_{2}^{n-1}$ & $V_{2}^{n}$ \\
\hline.. &.. &.. &.. &.. &.. \\
\hline$R B_{n-1}$ & $V_{n-1}^{1}$ & $V_{n-1}^{2}$ &.. & $V_{n-1}^{n-1}$ & $V_{n-1}^{n}$ \\
\hline$R B_{n}$ & $V_{n}^{1}$ & $V_{n}^{2}$ &.. & $V_{n}^{n-1}$ & $V_{n}^{n}$ \\
\hline \multicolumn{6}{|c}{ Table 1. RBs mapping with the proposed Scheduling Mechanism }
\end{tabular}

The utility value of each block can be considered as a specified class of QoS (6 class of QoS in the case of LTE-M due to its 6 RBs). Hence with the proposed mechanism, the devices with the most delay sensitive services are firstly served (multiperson video call, Autonomous driving, Real-time gaming, tactile internet, etc.), which will enhance the overall network QoS and maximize the bandwidth utilization.

\section{CONCLUSION}

We present in this paper a performance evaluation of LTE-M in terms of end-to-end delay and number of supported devices. Moreover, a novel scheduling mechanism is also proposed based on the optimization theory which enhances the QoS of M2M communications in terms of delay and increases the number of connected devices by maximizing the use of the available bandwidth.

\section{REFERENCES}

[1] Zheng, K., Ou, S., Alonso-Zarate, J., Dohler, M., Liu, F., \& Zhu, H. (2014). Challenges of massive access in highly dense LTE-advanced networks with machine-to-machine communications. IEEE Wireless Communications, 21(3), 12-18.

[2] Chin, W. H., Fan, Z., \& Haines, R. (2014). Emerging technologies and research challenges for $5 \mathrm{G}$ wireless networks. IEEE Wireless Communications, 21(2), 106-112.

[3] De Mattos, W. D., \& Gondim, P. R. (2016). M-Health Solutions Using 5G Networks and M2M Communications. IT Professional, 18(3), 24-29.

[4] EXALTED, EXpAnding LTE for Devices: Home. URL: http://www.ictexalted.eu/fileadmin/documents/EXALTED_WP2_D2.1.p df, Date of access 26.03.2012

[5] Tikhvinskiy, V., \& Bochechka, G. (2015). Prospects and QoS Requirements in $5 \mathrm{G}$ Networks. Journal of Telecommunications and Information Technology, (1), 23. 